\title{
Alzheimer - wo stehen wir jetzt?
}

Mit zunehmend besserer Diagnostik, sind scharfe Abgrenzungen zwischen vaskulärer und Alzheimer-Demenz nicht mehr möglich. Je früher eine Erkrankung behandelt wird, desto länger bleibt die Selbstständigkeit erhalten.

Die Epidemiologie spricht Bände - derzeit leiden hierzulande 112.000 Menschen an einer Form der Demenz, 70.000 an der Alzheimer-Demenz. Im Jahr 2050 werden es 234.000 sein.

Insbesondere bei der AlzheimerDemenz kommt es zu einer kontinuierlich fortschreitenden Beeinträchtigung, primär der Gedächtnisleistung. Später sind weitere kognitive Einbußen und schließlich Veränderungen der Persönlichkeit und des Verhaltens zu beobachten. Bei der primären Demenz ist das Organ Gehirn direkt betroffen, bei der sekundären Demenz liegt das schädigende Agens außerhalb des Gehirns. Reversible und irreversible Formen werden unterschieden, weitere Unterteilungen betreffen die Ätiologie und den Zeitpunkt des Auftretens (senil, präsenil).

Die Unterteilung in Alzheimer-Demenz mit primär degenerativer Genese durch pathologische Proteinakkumulation, vaskuläre Demenz, gemischte Demenz, frontotemporale Demenz und sonstige Formen lässt sich nur eingeschränkt aufrechterhalten. Mit zunehmend besserer Diagnostik, v. a. durch hoch auflösende Bildgebung, sind solche scharfen Abgrenzungen nicht mehr möglich, insbesondere jene zwischen vaskulärer Demenz und Alzheimer-Demenz.

Die Entscheidung im DSM-5, eine Veränderung der Nomenklatur vorzunehmen, war daher notwendig und konsequent. Hier spricht man nun von neurokognitiven Störungen (schwer/ leicht) mit oder ohne Verhaltensstörung, und es wird zusätzlich der Wahrscheinlichkeitsgrad der Ätiologie angegeben. Beispiel: 78-jährige Frau mit schwerer neurokognitiver Störung mit erheblicher Verhaltensstörung, basie- rend wahrscheinlich auf einer Alzheimer-Demenz.

\section{Letzte verbliebene Option}

Nach der Enttäuschung durch den Flop der Alzheimer-Impfung bleiben die Antidementiva als medikamentöse Therapieoption sowohl bezüglich der kognitiven Beeinträchtigungen als auch der Verhaltensveränderungen übrig. Je frühzeitiger eine beginnende Demenzerkrankung behandelt wird, desto länger bleibt die Selbstständigkeit erhalten, und somit können die Gesamtbehandlungskosten, vor allem in den ersten Jahren, deutlich reduziert werden (Jagsch 2014). Leitlinien und Konsensuspapiere stellen wichtige Informationsquellen und Entscheidungshilfen dar, z. B. Psychopharmakotherapie beim älteren Menschen.

Die Diagnostik und Differenzialdiagnostik der Demenzen sind weit fortgeschritten, spezifische Bio-Mar- ker können bereits in Vorstadien der Alzheimer-Demenz nachgewiesen werden, neben der MRT stehen Positronenemissionstomografen zur Verfügung, die in Zusammenschau mit der Klinik, der (Außen-)Anamnese, der neuropsychologischen Diagnostik und den Ergebnissen der unspezifischen Durchuntersuchung die Sensitivität und Spezifität der Diagnose Alzheimer-Demenz auf etwa $90 \%$ erhöhen (Fiedler et al. 2012). Dem gegenüber stehen die geringe Konsequenz bestimmter Untersuchungen für die Betroffenen neben dem Stress, den inflationäre Diagnostik oft bedeutet und die gesundheitsökonomische Perspektive.

psychopraxis. neuropraxis 2018 $\cdot 21: 49$ https://doi.org/10.1007/s00739018-0460-8

(c) Springer-Verlag GmbH Austria, ein Teil von Springer Nature 2018

\section{Buchtipp: Lebensqualität im Alter}

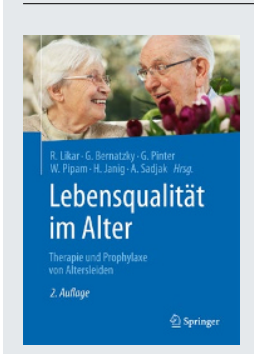

Dieses Buch setzt sich in einer aktualisierten Neuauflage mit dem Thema des Älterwerdens und den daraus resultierenden Änderungen der Befindlichkeit auseinander. Nach einleitenden Kapiteln über grundlegende Aspekte der Lebensqualität, Lebensstil und Bedürfnisse, demographische Prognosen, biologische Grundlagen des Alterns und über verschiedene im Alter oft auftretende Krankheitssymptome werden moderne Therapieformen häufiger Alterskrankheiten dargestellt. Weitere Kapitel sind der Behandlung der krankheits- und therapiebedingten Beschwerden und Nebenwirkungen und der Vorbeugung altersbedingter Leiden gewidmet. Ergänzt wurden in der Neuauflage Kapitel zur Verhaltenstherapie, Spiritualität, Gedächtnistraining, Harninkontinenz und Chemotherapie im Alter.

Lebensqualität im Alter

Therapie und Prophylaxe von Altersleiden

Likar R., Bernatzky G., Pinter G., Pipam W., Janig H., Sadjak A. (Hrsg.) Springer VS,

2. Auflage 2017, XXII, 422 Seiten, 43. Abb., Softcover: ISBN 978-3-662-53100-6; Preis: $€ 51,39$

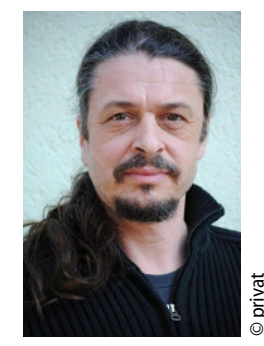

Mag. Dr. Herwig Oberlerchner

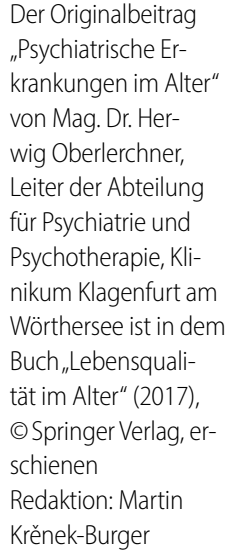

